\title{
RCPE UK Consensus Statement on Diabetes
}

\author{
Members of the Consensus Panel
}

doi:10.4997/JRCPE.20I0.S06

The prevalence of diabetes is increasing rapidly around the world as the population ages and become more obese. It is a condition that demands much of patients and diabetic control is often poor, particularly in the young. Treatment and care must take account of patients' individual needs and preferences. The increasing incidence of this disease will require additional resources being committed to proven services and treatments that deliver value for money.

\section{WHO CAN PREVENT DIABETES?}

Two thirds of the UK adult population is overweight (body mass index, $\mathrm{BMI}>25 \mathrm{~kg} / \mathrm{m}^{2}$ ) and one quarter obese $\left(\mathrm{BMl}>30 \mathrm{~kg} / \mathrm{m}^{2}\right)$, reflecting excess calorie intake and low levels of physical activity. Obesity is strongly associated with risk of developing type 2 diabetes, cardiovascular diseases and some cancers. A whole population approach to prevention is needed. This requires action by:

- People and society: Patients, their families and the community at large all have a role in preventing type 2 diabetes. This includes full participation of, and support for, those at highest risk, including some ethnic minority and economically deprived communities.

- Government and its institutions: Greater leadership is needed from Government to improve our obesogenic environment. The lessons from effective legislation on smoking should be used to promote healthier diets, increased physical activity and to inform transport and planning policy. The food and drink and catering industry should be more tightly regulated by legislation in the interests of public health. For example, restrictions on 'less healthy' food and drink advertising in children's television programmes should be extended to non-broadcast media and the wider marketing environment.

- NHS:The NHS has a crucial role to play in primary prevention and detection of diabetes through health promotion, advocacy, the training and education of its staff, community partnerships and opportunistic case finding among high-risk groups. There is insufficient evidence and too many uncertainties about the risks, practicalities, benefits and costs to support a national, population-based screening programme at this time. However, there is good evidence that lifestyle intervention in high-risk groups can prevent or delay the onset of type 2 diabetes, but translational research is required to define how to put these findings into everyday practice.

\section{WHAT ARE THE PRACTICAL IMPLICATIONS OF DEVELOPMENTS IN GENETICS?}

Current knowledge on the application of genetics suggests the following:

- There is no evidence that general population genetic screening for diabetes is beneficial and it is not recommended.

- Genetic testing of patients at increased clinical risk of diabetes provides little additional predictive value and is not recommended.

- As current clinical diagnostic criteria misclassify a small proportion of patients, there is a role for testing for monogenic diabetes in selected patients, as they may benefit from alternative treatment; for example, hyperglycaemia with onset before six months of age and young onset type 2 diabetes with a strong family history. This is an evolving field and it is difficult to define clear criteria in other groups of patients (see www.diabetesgenes.org).

- The resource implications of genetic profiling are not defined and require further research.

Research on genetic testing shows promise in elucidating disease mechanisms and developing new treatments.

\section{WHICH PSYCHOLOGICAL INTERVENTIONS WORK?}

- Improving diabetes health will only occur if the individual's health beliefs, health-related behaviours, knowledge and self-care skills and their personal circumstances are considered and supported.

- The organisation of diabetes healthcare in the UK inhibits sufficient focus on these issues.

- Previous work has demonstrated the theoretical principles of psychological interventions such as behaviour modification, motivational interviewing, cognitive behavioural therapy goal-setting and coping skills (e.g. SIGN II6). Robust, high-quality research to assess their generalisability, cultural suitability, applicability and their implementation across populations and between different disease groups is required.

- Appropriate psychosocial and educational services tailored to the individual's circumstances should be available and be offered by an appropriately trained healthcare provider.

- The training and continuing education of all those involved in the care of patients should be informed by applied psychology and include the need for a person-centred approach. 


\section{WHAT AFTER METFORMIN?}

If agreed treatment goals cannot be attained on a combination of lifestyle modification and metformin, a review of the following should occur:

- The patient's ability to achieve suggested lifestyle modification and adherence to metformin treatment following appropriate reinforcement and support.

- The appropriateness of the treatment goal for the individual, taking into account their personal preferences, occupation, co-morbidities and likely ability to adhere to more complex treatment regimens.

Additional pharmacological treatment should then be considered if necessary. The consensus is to follow SIGN or NICE guidance. There are no randomised controlled trials to definitively determine the effect of combination therapy on clinically important outcomes. Individual clinician judgement and expertise should still be applied to the needs and circumstances of individual patients and should be documented and justified.

The selection of new drugs or new combination regimens should not be based on the $\mathrm{HbA}_{\mathrm{lc}}$ level and weight change alone but also on other factors, including hypoglycaemia, quality of life and cardiovascular disease.

A range of research studies are required on long-term outcomes and late-onset adverse effects of pharmaceuticals on a population basis. Further research on patient concordance with medication and lifestyle interventions should be undertaken.

\section{WHAT ARE THE BEST MODELS OF CARE FOR CHILDREN AND ADOLESCENTS?}

There is no evidence that one model of care is better than any other. The best performing teams appear to have at their core person-centred attitudes and the ability to motivate the young person and their families and are teams that can be trusted to deliver at all times.

Care for this group of people is usually provided by specialists and should be provided by multidisciplinary teams, including physicians, specialist nurses, dieticians and psychological support workers. These teams should be resourced to provide the full range of services required - both technical (e.g. insulin pumps) and supportive.

Key elements of care, specifically tailored to the needs of young people, include:

- Health system - resources, integrated structures and planning, regional and local networks.

- Delivery systems - accessible service; multidisciplinary team, adequate frequency of consultations, audit and governance.

- Decision support - clinical care consistent with best evidence and patient preference, delivery of relevant information and planned transition to adult services.

- Self-management support - shared goals, sustained relationships, understanding of responsibilities, requisite skills and school support.

- Clinical information systems - databases, recall, audit and research.

- A patient-focused, goal-led approach, audited against robust quality standards.

The importance of social networking, peer support, family support and sustained rapport with professionals has been demonstrated in some settings and requires further research. 